\title{
LA EFICIENCIA EN FORMA DÉBIL Y EL PODER PREDICTIVO DE LOS MODELOS ARMA-GARCH
}

\author{
Adrián Hernández-del-Valle* \\ Escuela Superior de Economía, Instituto Politécnico Nacional \\ Federico Reina Sosa \\ Departamento de Posgrado e Investigación, Instituto Politécnico Nacional
}

\section{Héctor Allier Campuzano}

\author{
Escuela Superior de Economía, Instituto Politécnico Nacional
}

(Recibido 6 de noviembre 2002, aceptado 20 de febrero 2003)

\section{Fiesumen}

Un concepto importante en la literatura financiera que trata acerca de la modelación y el pronóstico de series de tiempo de los precios de activos es el de eficiencia en forma débil. Aplicamos una prueba de eficiencia en forma débil a una muestra de series de tiempo de los precios de acciones que cotizan en la Bolsa Mexicana de Valores (BMV) entre los años de 1991 y 2000. Utilizamos un modelo ARMA-GARCH, con series de diferente frecuencia y extrapolamos a diferentes intervalos de tiempo, para probar el poder predictivo ex ante de los modelos. Reportamos resultados acerca de las series que generan el mejor pronóstico, así como su error promedio e intervalo de error.

\section{Abstract}

An important concept in the financial literature dealing with the modeling and forecasting of financial time series is that of weak efficiency. We apply a test of weak efficiency to a sample of securities of the Mexican Stock Market between 1991-2000. We use ARMA-GARCH modeling with series of different frequency and extrapolate from different time intervals to prove the $E x$ ante predictive power of the models. We report results about those time series that generate the best forecast, as well as its average error and error interval.

Clasificación JEL: G14, C32

Palabras clave: Mercados eficientes, Series de Tiempo

* Escuela Superior de Economía. Plan de Agua Prieta, No. 66, Edif. "C". Col. Plutarco Elías Calles (Santo Tomás), C.P. 11340. México, D.F. Teléfono: 5586-3534 y 5729-6000 (ext. 62012 y 62036). Correo electrónico: ahdv@hotmail.com

Los autores desean agradecer a dos dictaminadores anónimos sus valiosas observaciones. 


\section{Introducción}

Desde el origen de los mercados financieros ha existido el interés por hacer pronósticos de los precios y rendimientos de los activos disponibles en dichos mercados. En este contexto, uno de los conceptos que más ha influido en el desarrollo de la literatura financiera es el de eficiencia en forma débil. Fama (1972) proporcionó una clasificación de los diferentes tipos de eficiencia, argumentando que los mercados pueden ser eficientes en tres niveles según la información reflejada en los precios. En el concepto de eficiencia en forma débil, el precio actual refleja toda la información contenida en la historia de la serie. En el concepto de eficiencia en forma semi-fuerte, el precio actual refleja no sólo toda la información histórica de los precios, sino toda la información pública (los estados financieros y noticias). Finalmente, la eficiencia en forma fuerte implica que el precio actual refleja toda la información, tanto pública como privada (confidencial).

La utilidad de la noción de eficiencia en forma débil es que si la serie de tiempo de precios o rendimientos accionarios es no correlacionada serialmente y homoscedástica, cuando se modela como autorregresiva de primer orden, AR(1), en el tiempo $t$, entonces basta usar el precio o el rendimiento de la serie en el tiempo $t$, el presente, para pronosticar el precio o rendimiento en el tiempo $t+1$, el futuro. Si por el contrario, la serie de tiempo guarda autocorrelación serial de orden $n$ o es heteroscedástica, eso nos indica que existe información en la serie de los precios, en sus residuos o en sus varianzas que debe incorporarse al modelo para incrementar su poder predictivo.

El proceso que condujo a la noción de eficiencia tiene una larga historia, que inicia a principios de 1930 con los trabajos de Cowles (1933), Cowles y Jones (1937), Kendall (1953) y Working (1960), en donde se llegó a la "triste" conclusión de que no se pueden predecir cambios en los precios con base en cambios pasados de las series. Motivados por estos primeros artículos aparecieron otros trabajos que investigaron la hipótesis de que los cambios en los precios son independientes. A tal hipótesis se le denominó caminata aleatoria. La eficiencia en forma débil es la que se asocia con esta hipótesis.

Este trabajo presenta tres resultados principales. Primero, la metodología para la construcción de modelos de pronóstico de precios accionarios consiste en: correr las series de tiempo accionarias como modelos autorregresivos de primer orden, AR(1). Si el modelo resulta no correlacionado serialmente y homoscedástico, se concluye que la serie es eficiente en forma débil y se construyen los pronósticos. Si el modelo no cumple las dos condiciones estipuladas, entonces se dice ineficiente y se corrige mediante modelación ARMA-GARCH.

En este contexto, las series accionarias de la Bolsa Mexicana de Valores muestran ineficiencia y heteroscedasticidad, que tiende a "desaparecer" conforme se reduce la longitud de las series. Cuando se usaron datos diarios a lo largo de un periodo de ocho años, 1991-1999, 70.96\% de las empresas en la muestra resultaron ineficientes y $90.32 \%$ heteroscedásticas. En comparación con $9.67 \%$ ineficientes y $16.12 \%$ heteroscedásticas cuando se usaron datos trimestrales.

Segundo, el pronóstico puntual es imposible, de tal forma que el poder predictivo ex ante de los modelos está determinado por su error medio y por la 
longitud de los intervalos de confianza; los cuales, se espera, que sean lo más pequeño posible. Con base en esto, los modelos estimados, tomando series de un año de longitud producen los mejores pronósticos a un año, seis, tres y un mes; mientras que modelos hechos con 6 meses de datos producen mejores resultados a 15 días y una semana. En este último caso, el error medio del pronóstico a una semana fue de $\$ 0.28$ y la longitud del intervalo, al $90 \%$ de confianza estadística, de \$1.81. Esto nos permite afirmar que aunque la eficiencia en forma débil merma el poder predictivo ex ante de los modelos ARMA-GARCH, en el sentido de que impide el pronóstico puntual, aún nos permite hacer pronósticos bastante acertados. Finalmente, en concordancia con el estudio de Loughran y Ritter (2000), se encontró que es más factible hallar ineficiencias, y en consecuencia oportunidades de inversión, fuera de la muestra del IPC.

El presente trabajo de investigación está dividido en cinco secciones. En la sección 2 se considera el contexto económico. El la sección 3 se explica la definición de eficiencia y se describen las herramientas técnicas necesarias para su análisis. Los modelos autorregresivos de medias móviles, ARMA, y los modelos autorregresivos de heteroscedasticidad condicional generalizados, GARCH. En la sección 4 se presentan los resultados de las diferentes pruebas aplicadas a las series de tiempo accionarias de la Bolsa Mexicana de Valores. Por último, en la sección 5 se exponen las conclusiones y se incluyen algunos anexos sobre el análisis empírico.

\section{El contexto de México}

La literatura en torno al análisis de la eficiencia en forma débil es vasta dada la simplicidad y aplicabilidad del concepto. Sin embargo, en México se ha publicado poco acerca del tema (Ludlow (1997) y Moctezuma (1998)). Este último, analiza la eficiencia en forma débil de la serie de tiempo de los rendimientos diarios, entre 1989 y 1997, y de los rendimientos de cada 15 minutos, en el último trimestre de 1997, del Indice de Precios y Cotizaciones de la BMV. En ambos casos, el autor usa una modelación ARMA-GARCH y aplica pruebas no paranétricas sobre las corridas. Con base en su análisis, Moctezuma (1998) concluye que en el caso de los rendimientos diarios, existe evidencia en contra de la hipótesis de eficiencia en forma débil, ya que para determinar el movimiento de los precios puede utilizarse información de los rendimientos observados en los últimos dos días, o de la volatilidad en los últimos tres días. Asimismo, en el caso de los rendimientos de cada 15 minutos también encontró evidencia que sustenta la ineficiencia en forma débil. De acuerdo, con el modelo que el autor estimó para definir la trayectoria de los precios durante los siguientes 15 minutos, un inversionista debe hacer uso de-la información de las últimas seis horas.

Aunque el trabajo de Moctezuma (1998) es de gran claridad, deja algunas preguntas sin contestar. Por ejemplo, no incluyó en su muestra a empresas que estuvieran fuera del IPC. Lo cual de cierta forma resulta ser lógico, debido a que se trata de empresas pequeñas con poca bursatilidad y capitalización. Sin embargo, esto contradice la afirmación de Loughran y Ritter (2000) acerca de que la probabilidad de encontrar valuaciones incorrectas (i.e. ineficiencias y en consecuencia oportunidades de inversión) es mayor en empresas pequeñas, precisamente aquellas que no están incluidas en los índices, que en empresas 
grandes. Además, Moctezuma (1998) no proporciona información acerca del poder predictivo ex ante de los modelos que estimó. Por ejemplo, su resultado acerca de la volatilidad para los rendimientos diarios corresponde a un modelo autorregresivo de heteroscedasticidad condicional de tercer orden, $\operatorname{ARCH}(3)$. Sin embargo, Corhay y Rad (1991), Chou (1988) y Poon y Taylor (1992) encontraron que el modelo autorregresivo de heteroscedasticidad condicional generalizado, GARCH $(1,1)$, no sólo es suficiente, sino que con frecuencia proporciona el mejor ajuste econométrico. Finalmente, el autor nos proporciona un modelo agregado del IPC. No obstante, hacer uso de esas ineficiencias implicaría una estrategia que requiere mucha información, información que cuesta y que toma tiempo recolectar, probablemente un tiempo mayor que la duración de la ineficiencia misma. Así pues, aunque el resultado es interesante es de poca utilidad, porque sería excesivamente costoso para los inversionistas reproducir la estrategia de inversión generadora de rendimientos extraordinarios haciendo uso de esas ineficiencias.

Con base en las observaciones anteriores, el objetivo de este trabajo es, primero, determinar una metodología para la construcción de modelos econométricos con un alto poder predictivo ex ante. Una consecuencia inmediata de este primer objetivo, fue la realización de un análisis de la eficiencia en forma débil sobre los precios de cierre diarios de una muestra de 31 empresas, la cual incluyó empresas muestreadas en el IPC y empresas que no lo están. Segundo, determinar los modelos con el mayor poder predictivo ex ante. Finalmente, se hacen generalizaciones en torno a la relación que guarda la eficiencia y el poder predictivo de los modelos. Para lo cual se usó un modelo ARMA-GARCH y se emplearon series de tiempo de diferente longitud. En cada caso, se excluyó el último año de datos para poder probar el poder predictivo ex ante del modelo; variando, también, la longitud de los pronósticos. Es decir, pronósticos a un año, a seis, a tres y a un mes, a 15 días y a una semana.

\section{Eficiencia del mercado}

\section{1 ¿Qué es un mercado eficiente?}

Econométricamente diremos que una serie de tiempo accionaria es eficiente si se cumple la propiedad de Markov. Formalmente, la propiedad de Markov de un proceso estocástico $\left\{X_{0}, X_{1}, \ldots\right\}$ se expresa de la siguiente manera:

$$
P\left(X_{t+1}=p_{t+1} \mid X_{0}=p_{0}, \ldots, X_{t}=p_{t}\right)=P\left(X_{t+1}=p_{t+1} \mid X_{t}=p_{t}\right),
$$

es decir, dada la historia del proceso hasta el presente, $X_{0}=p_{0}, \ldots, X_{t}=p_{t}$, la probabilidad de que la variable aleatoria $X$ tome el valor de $p_{t+1}$ depende sólo de su presente $X_{t}=p_{t}$. La propiedad de Markov es una condición frecuentemente observable en fenómenos reales, ya sean físicos, biológicos o económicos.

El ejemplo más sencillo de un proceso de Markov, y que es precisamente el que usó Fama (1972) para describir la eficiencia de mercado, es el de la caminata aleatoria. Dicho proceso, que denotaremos por $\left\{p_{t}\right\}$, está determinado por:

$$
p_{t}+p_{t-1}=\varepsilon_{t} \text { para } t=1,2, \ldots,
$$

en donde $p_{0}$ es el "estado inicial", una variable aleatoria dada, y las "perturbaciones" $\varepsilon_{t}$ son variables aleatorias independientes. Supondremos que $\mathrm{E}\left(\varepsilon_{t}\right)=0$ y $\mathrm{E}\left(\varepsilon_{t}^{2}\right)=\sigma^{2}$. 
Econométricamente, la caminata aleatoria es un modelo autorregresivo de primer orden, AR(1), estacionario:

$$
p_{t}=\alpha_{1} p_{t-1}+\varepsilon_{t}
$$

La componente estacionaria se da si $\left|\alpha_{1}\right|<1$. Sin embargo, existe debate respecto al hecho de exigir que el modelo sea estacionario. Para autores como Mills (1999), la hipótesis de la caminata aleatoria resulta demasiado restrictiva. Otro problema es que si se permite que $\alpha_{1}$ sea mayor o igual a 1 , entonces la variancia se indetermina o resulta compleja. Con base en lo anterior, Mills (1999) propone el siguiente modelo alternativo

$$
p_{t}=(1+\delta) p_{t-1}+\varepsilon_{t}, \text { con } \delta>0
$$

La diferencia entre ambos modelos radica, en que el segundo considera la existencia de una tasa de rendimiento, $\delta$, lo cual obliga a que los precios se muevan de manera aleatoria, pero dentro de una tendencia. Como se puede observar, el modelo propuesto por dicho autor es precisamente una submartingala.

\subsection{Modelos para el pronóstico de series de tiempo financieras}

El objetivo, ahora, es responder a las siguientes preguntas. ¿Cómo reconocer econométricamente que el mercado es ineficiente? y ¿cómo modelar las ineficiencias? En la sección anterior se definió lo que es la eficiencia, pero ¿cuáles son las implicaciones de la ineficiencia del mercado para el inversionista?

Básicamente, la idea es que si el mercado no es eficiente, entonces se puede usar la información pasada de las series de tiempo accionarias para pronosticar el futuro; con lo cual, se puede conocer el precio de la acción con días de anticipación, y a partir de eso se sabrá cuándo vender y cuándo comprar. Para la modelación econométrica de las series de tiempo bajo ineficiencia, se usan los modelos autorregresivos de medias móviles, ARMA, y los de heteroscedasticidad condicional generalizados, GARCH.

\subsubsection{Modelos autorregresivos de medias móviles, ARMA}

Como se pudo observar en la sección anterior, la eficiencia en forma débil de un mercado accionario se caracteriza por el hecho de que sus series de tiempo financieras cumplen la propiedad de Markov, y el mejor estimador en el momento siguiente es el valor presente de la serie. ¿Qué sucede si el mercado es ineficiente? ¿Cómo se le reconoce?

Si el mercado es eficiente, entonces al modelarlo como autorregresivo de primer orden, $\operatorname{AR}(1)$, las $p_{t}$ deberán ser estadísticamente independientes, i.e. sus términos de error, $\varepsilon_{t}$, deben ser ruido blanco. De no cumplirse esta propiedad los modelos estimados serían, en teoría, inapropiados y los pronósticos tendrían errores. Si las $p_{t}$ no son independientes se deben usar valores pasados de la variable de la serie de tiempo y/o valores pasados de los términos de error. La metodología Box-Jenkins permite hacer lo anterior.

El enfoque Box-Jenkins, para los modelos autorregresivos de medias móviles, ARMA, consiste en extraer los movimientos predecibles, o con patrón, de la información observada a través de una serie de iteraciones. Primero, se trata 
de identificar la serie de tiempo dentro de una clase de modelos lineales, AR(1). Después, se evalúa el modelo escogido contra la información histórica para ver si describe el proceso generador subyacente de la serie. Se dice que el modelo se ajusta bien, si las diferencias entre los valores estimados y los observados son pequeñas, independientes y aleatorias.

Además, para el caso particular de la predicción, se espera obtener coeficientes de determinación altos. En otras palabras, $R^{2}$ y $\bar{R}^{2}>0.90$. Si el modelo muestra autocorrelación serial en los residuos, usaremos modelos de medias móviles de orden $q$, MA $(q)$. En otro caso, usaremos modelos autorregresivos de orden $p, A R(p)$.

\subsubsection{Modelo autorregresivo de primer orden}

Una serie de tiempo (o proceso estocástico) de la forma

$$
p_{t+1}=p_{t}+\varepsilon_{t+1}
$$

se dice que es un proceso autorregresivo de primer orden, AR(1). En este caso, supondremos que las perturbaciones aleatorias, $\varepsilon_{t}$, son independientes y con $E\left(\varepsilon_{t}\right)=0$. Asimismo, a menos que se diga lo contrario, supondremos que $P_{0}$ es independiente de $\varepsilon_{t}$ para todo $t$.

Como se puede observar, $p_{t+1}=p_{t}+\varepsilon_{t+1}$ es precisamente la "caminata aleatoria" que ya mencionamos en la sección 1. Si la propiedad de Markov no se cumple, entonces se dirá que el mercado es ineficiente en forma débil. La implicación inmediata de este hecho es que la serie de tiempo se podría modelar con base en su historia (incluyendo elementos autorregresivos de orden superior, $A R(p)$ ), con base en los residuos rezagados (incorporando elementos de medias móviles de orden superior $\mathrm{MA}(q))$ y/o con base en la información contenida en la varianza o sus residuos (elementos autorregresivos heteroscedásticos, ARCH, o autorregresivos heteroscedásticos generalizados, GARCH).

\subsubsection{2 $\operatorname{AR}(p)$}

Se dice que la serie $p_{t}$ es autorregresiva de orden $p$, si en cada tiempo $t, p_{t}$ es función de $p$ valores rezagados de la misma serie, i.e.

$$
p_{t}=\alpha_{0}+\alpha_{1} p_{t-1}+\alpha_{2} p_{t-2}+\ldots+\alpha_{p} p_{t-p}+\varepsilon_{t}
$$

\subsubsection{3 $\mathrm{MA}(q)$}

Un modelo de media móvil es un promedio ponderado de errores estocásticos pasados. Es decir, $p_{t}$ es una media móvil de primer orden, MA(1), si es función del error del periodo anterior, i.e.

$$
p_{t}=\alpha_{0}+\varepsilon_{t}+\beta_{1} \varepsilon_{t-1}
$$

y de orden $q, \operatorname{MA}(q)$, si es función de $q$ errores rezagados, es decir,

$$
p_{t}=\alpha_{0}+\varepsilon_{t}+\beta_{1} \varepsilon_{t-1}+\beta_{2} \varepsilon_{t-2}+\ldots+\beta_{q} \varepsilon_{t-q} .
$$




\subsubsection{Modelos GARCH}

Generalmente, cuando se trabaja con series de tiempo financieras se espera que la varianza de las perturbaciones, $\varepsilon_{t}$, dependa de la volatilidad pasada, la cual está asociada a una gran cantidad de periodos rezagados debido a que se emplean datos diarios, semanales o intradía. Además, se acepta que la volatilidad no es constante, sino que tiende a oscilar entre periodos de alta y de baja variación. Por lo anterior, los modelos ARCH, introducidos por Engle (1982), resultan de gran utilidad.

La variación sistemática de la volatilidad de los rendimientos a lo largo del tiempo implica que la varianza en $t$ se puede dividir en dos componentes, uno predecible y otro impredecible. A el componente predecible se le llama la varianza condicional y está en función de toda la información disponible hasta el tiempo $t-1$. Esta información puede incluir variables exclusivas de la empresa, de toda la economía y de la historia pasada de la serie de rendimientos. Es claro que modelar la varianza condicional de los rendimientos de las acciones es importante porque la volatilidad esperada es un elemento fundamental de la decisión sobre la selección de portafolios, así como en la modelación de los precios de acciones.

El modelo univariado ARCH, en su forma más general (Engle, 1982), toma la varianza condicional, $\sigma_{t}$, en el tiempo $t$, como función de variables rezagadas exógenas y endógenas, del vector de parámetros, de residuos pasados y del tiempo mismo.

\subsubsection{ARCH(1)}

La varianza del error estocástico, $\mathrm{E}\left(\varepsilon_{t}^{2}\right)=\sigma_{t}^{2}$, depende de la volatilidad del último periodo,

$$
\sigma_{t}^{2}=\alpha_{0}+\alpha_{1} \varepsilon_{t-1}^{2}
$$

\subsubsection{2 $\operatorname{ARCH}(p)$}

El modelo anterior se puede generalizar para $p$ periodos de la siguiente manera,

$$
\sigma_{t}^{2}=\alpha_{0}+\alpha_{1} \varepsilon_{t-1}^{2}+\alpha_{2} \varepsilon_{t-2}^{2}+\ldots+\alpha_{p} \varepsilon_{t-p}^{2}
$$

Una dificultad práctica de los modelos ARCH es que para un número grande de periodos, $p$, la estimación lleva frecuentemente a la violación de la restricción de no negatividad de los coeficientes $\alpha_{i}$, la cual se necesita para asegurar que la varianza condicional, $\sigma_{t}^{2}$, será siempre positiva. En las primeras aplicaciones de este modelo, se imponía una estructura de rezagos decreciente sobre $\operatorname{los} \alpha_{i}$ para asegurar que se cumpliera la restricción. Sin embargo, para tener una mayor flexibilidad Bollerslev $(1986,1988)$ propuso una extensión del ARCH: el ARCH generalizado (GARCH).

\subsubsection{GARCH $(1,1)$}

Modelo de heteroscedasticidad condicional autorregresivo generalizado.

$$
\sigma_{t}^{2}=\alpha_{0}+\alpha_{1} \varepsilon_{t-1}^{2}+\lambda_{1} \sigma_{t-1}^{2}
$$


En este caso, la varianza del error estocástico tiene tres componentes: una constante, la volatilidad del último periodo (el término $\mathrm{ARCH}(1)$ ) y la varianza del último periodo (el término $\mathrm{G}(1)$ ). En general, se puede tener cualquier número de términos ARCH y cualquier número de términos GARCH.

\subsubsection{4 $\operatorname{GARCH}(p, q)$}

El modelo $\operatorname{GARCH}(p, q)$ se refiere a la siguiente ecuación para $\sigma_{t}^{2}$ :

$$
\sigma_{t}^{2}=\alpha_{0}+\alpha_{1} \varepsilon_{t-1}^{2}+\ldots+\alpha_{p} \varepsilon_{t-p}^{2}+\lambda_{1} \sigma_{t-1}^{2}+\ldots+\lambda_{q} \sigma_{t-q}^{2} .
$$

A partir de esta descripción técnica de las herramientas econométricas, la siguiente sección presenta la evaluación de la eficiencia en forma débil de la serie financiera de la Bolsa Mexicana de Valores para el periodo entre los años de 1991-2000.

\section{El caso de las acciones de la BMV (1991-2000)}

EI objetivo de esta sección es probar el poder predictivo de los modelos ARMAGARCH aplicados a series de tiempo accionarias de la BMV. Para ello, es necesario primero probar la hipótesis de eficiencia en el mercado accionario mexicano. Es decir ¿las series de tiempo accionarias de la BMV, cumplen con la propiedad de Markov? ¿Se pueden modelar como AR(1)?

En segundo lugar se deben determinar las características óptimas de las series para poder construir modelos con alto poder predictivo, en concreto ¿Series de cuánto tiempo son recomendables para construir los modelos con el mejor poder predictivo? En este proceso, lo que deseamos encontrar es lo siguiente: 1) homoscedasticidad, 2) no existencia de correlación serial, 3) significancia de parámetros y 4) el modelo más sencillo posible que cumpla las características 1,2 y 3.

\subsection{La muestra}

La muestra incluye a 31 empresas mexicanas, de las cuales solo diecisiete de ellas están incorporadas dentro de la serie del IPC (hasta julio de 2000). Se emplearon series de tiempo con la historia más larga (fecha de inicio, el 2 de diciembre de 1991) lo cual representa alrededor de 2289 datos. La base de datos fue Economática. Finalmente, para probar el poder predictivo ex ante de los modelos, se tomó el último año de datos de la serie de tiempo de cada empresa fuera de la muestra. Así se pudo comparar el pronóstico de los modelos con la realidad.

\subsection{Resultados de la prueba de eficiencia ¿las series son AR(1)?}

Se procedió de la siguiente manera. Primero, se excluyó el último año de datos de las series de tiempo de las empresas seleccionadas para evaluar el poder predictivo ex ante de los modelos ARMA-GARCH. Segundo, se corrieron las series de tiempo accionarias como modelos $\mathrm{AR}(1)$ en cuatro lapsos de tiempo distintos. Estos diferentes cuatro lapsos son: toda la serie (1991-1999); un año de datos (1998-1999); seis meses de datos (mayo-junio 1999) y tres meses de datos (agosto-noviembre 1999). Se dirá que existe eficiencia si los modelos 
AR(1) no presentan correlación serial. Para probar tal hipótesis se aplicaron las pruebas Durbin-Watson y Breusch-Godfrey.

Tabla 1. Las empresas seleccionadas.

\begin{tabular}{|c|c|}
\hline Fuera del IPC & Dentro del IPC \\
\hline Accel S.A. serie B & Alfa S.A. serie A, \\
\hline Gpo. Palacio de Hierro serie 1 & Apasco S.A. \\
\hline Cemex S.A. serie A & Banamex Accival GF serie O \\
\hline Continental Gpo. & Bimbo Gpo. serie A \\
\hline Cydsa S.A. serie A & DESC serie B \\
\hline Ekco & FEMSA UBD \\
\hline Gpo. Salinas y Rocha serie B & Grupo Carso serie A1 \\
\hline Gpo. Santander GF serie B & Gpo. Industrial Saltillo serie B \\
\hline Patria Reasegurador serie B & Kimberly Clark Mex. serie A \\
\hline Pepsigx (Gemex) serie B & Peñoles Industrias \\
\hline San Luis corp. serie A & Savia A \\
\hline Sidek Gpo. serie B & Soriana Organización serie B \\
\hline Situr Grupo serie B & Telmex serie L \\
\hline Transp. Marítima Mex. serie A & Televisa Gpo. Serie CPO \\
\hline & Vitro serie A \\
\hline & Walmart serie V \\
\hline & Tubos de Acero Mex. \\
\hline
\end{tabular}

Una prueba adicional es la de la existencia de homoscedasticidad. Si el modelo pasa esta prueba junto con la de no autocorrelación serial, entonces se dice que el mercado es eficiente en forma débil. Finalmente, considerando la probabilidad de la prueba ARCH-LM > 0.05 podemos decir que el modelo es homoscedástico; en caso contrario será necesario modelar la variancia condicional mediante GARCH. En la situación donde los AR(1) estimados presenten correlación serial y homoscedasticidad, se ajustarán mediante un modelo ARMA-GARCH.

En tercer lugar, se hacen pronósticos con los modelos estimados y corregidos para los cuatro lapsos de tiempo señalados. Lo anterior para poder responder las siguientes preguntas: ¿es eficiente en forma débil el mercado accionario mexicano? ¿De qué manera afecta la longitud de la serie de tiempo estimada a la eficiencia? ¿En dónde es más factible hallar ineficiencias y, por tanto, oportunidades de inversión? ¿Cuál es el poder predictivo ex ante de los modelos ARMA-GARCH? ¿Qué longitud debe tener la serie de tiempo para generan modelos con el mayor poder predictivo? ¿Cuál es el error medio y el intervalo de confianza de los pronósticos para los diferentes periodos usando los diferentes lapsos de estimación? y ¿la eficiencia merma el poder predictivo de los modelos?

Los resultados de la aplicación del modelo autorregresivo de primer orden, AR(1), a las series de tiempo de los precios de cierre diarios para el periodo 1991-1999 de la empresas mencionadas son: 
Tabla 2. Modelos usando toda la serie de datos: 1991-1999.

\begin{tabular}{|c|c|c|c|c|c|c|}
\hline Empresa & $\bar{R}{ }^{2}$ & Durbin-Watson & $\begin{array}{c}\text { Probabilidad } \\
\text { Breusch-Godfrey } \\
\text { correlación serial }\end{array}$ & $\begin{array}{c}\text { Coef. } \\
\alpha_{1}\end{array}$ & $\begin{array}{c}\text { Probabilidad } \\
\text { ARCH-LM }\end{array}$ & ¿Eficiencia? \\
\hline Accel & 0.997835 & 1.938726 & 0.020149 & 0.999867 & 0.026238 & No \\
\hline Palacio de Hierro & 0.993703 & 1.835340 & 0.000010 & 1.000085 & 0.790413 & No \\
\hline Cemex & 0.996392 & 1.733809 & 0.000000 & 1.000417 & 0.006823 & No \\
\hline Gpo. Continental & 0.998111 & 1.850388 & 0.000007 & 1.000115 & 0.000000 & No \\
\hline Cydsa & 0.994920 & 1.765462 & 0.000000 & 0.999602 & 0.000000 & No \\
\hline Ekco & 0.997434 & 1.771763 & 0.000000 & 0.999422 & 0.016578 & No \\
\hline Gpo. S\&Rocha & 0.998296 & 1.805847 & 0.000007 & 0.999091 & 0.767639 & No \\
\hline GF Santander & 0.999297 & 1.706844 & 0.000000 & 0.999629 & 0.000000 & No \\
\hline Patria & 0.496562 & 2.027866 & 0.671832 & 1.007723 & 0.989978 & Sí \\
\hline Pepsigx & 0.996027 & 1.900571 & 0.066371 & 1.000189 & 0.000000 & Sí \\
\hline San Luis Corp. & 0.998443 & 2.223375 & 0.000000 & 1.000112 & 0.000000 & No \\
\hline Sidek & 0.998180 & 1.762139 & 0.000001 & 0.999484 & 0.000000 & No \\
\hline Situr & 0.998052 & 1.642402 & 0.000000 & 0.999393 & 0.000000 & No \\
\hline TMM & 0.993180 & 1.907474 & 0.080207 & 1.000503 & 0.007808 & Sí \\
\hline Alfa & 0.998042 & 1.699914 & 0.000000 & 0.999811 & 0.000000 & No \\
\hline Apasco & 0.996707 & 2.090096 & 0.088805 & 1.000195 & 0.000000 & Sí \\
\hline Banamex & 0.993979 & 1.754458 & 0.000000 & 1.000813 & 0.000000 & No \\
\hline
\end{tabular}


Tabla 2. Modelos usando toda la serie de datos: 1991-1999

(continuación).

\begin{tabular}{|c|c|c|c|c|c|c|}
\hline Empresa & $\bar{R}^{2}$ & Durbin-Watson & $\begin{array}{c}\text { Probabilidad } \\
\text { Breusch-Godfrey } \\
\text { correlación serial }\end{array}$ & $\begin{array}{c}\text { Coef. } \\
\alpha_{1}\end{array}$ & $\begin{array}{c}\text { Probabilidad } \\
\text { ARCH-LM }\end{array}$ & iEficiencia? \\
\hline Bimbo & 0.997917 & 1.956785 & 0.103934 & 1.000020 & 0.000000 & Sí \\
\hline DESC & 0.997367 & 1.880082 & 0.009825 & 0.999787 & 0.000000 & No \\
\hline FEMSA & 0.998130 & 1.765886 & 0.000000 & 1.000598 & 0.000000 & No \\
\hline Gpo. Carso & 0.993739 & 1.825725 & 0.000092 & 0.999762 & 0.046184 & No \\
\hline Gpo. Ind. Saltillo & 0.998350 & 1.848812 & 0.000303 & 0.999897 & 0.000000 & No \\
\hline Kimberly Clark & 0.997590 & 1.943230 & 0.127221 & 1.000030 & 0.000000 & Sí \\
\hline Peñoles Ind. & 0.997976 & 1.824750 & 0.000052 & 0.999839 & 0.000000 & No \\
\hline Savia & 0.999119 & 1.782263 & 0.000001 & 1.000185 & 0.000000 & No \\
\hline Soriana & 0.998266 & 1.947948 & 0.061800 & 1.000350 & 0.000000 & Sí \\
\hline Telmex & 0.998283 & 1.710696 & 0.000000 & 1.000918 & 0.000000 & No \\
\hline Televisa & 0.996365 & 1.821285 & 0.000002 & 1.000569 & 0.000000 & No \\
\hline Tubos de Acero & 0.997913 & 1.732931 & 0.000000 & 1.000319 & 0.000000 & No \\
\hline Vitro & 0.992798 & 1.984500 & 0.229377 & 0.999509 & 0.000000 & Sí \\
\hline Walmart & 0.996672 & 1.929812 & 0.094881 & 1.000531 & 0.000000 & Sí \\
\hline
\end{tabular}




\subsection{Resultados de la corrección econométrica de los modelos}

Como se mencionó, las pruebas Durbin-Watson y Breusch-Godfrey revelan si existe autocorrelación serial en los residuos y/o en los valores rezagados de la serie de tiempo. Las funciones de autocorrelación (ACF) y de autocorrelación partial (PACF) que se observan en los correlogramas nos indican la magnitud del rezago. Por otro lado, la prueba ARCH-LM nos indica si existe homoscedasticidad, es decir, si la variancia es constante.

A continuación se presentan los resultados de la corrección econométrica hecha para las series de tiempo de aquellas empresas con precios ineficientes, a través del uso de un modelo ARMA-GARCH. La información se interpreta de la siguiente manera, por ejemplo: la serie de tiempo de los precios diarios de cierre de Accel, usando 8 años de datos (1991-1999), corresponde a un modelo AR(1) y GARCH $(1,2)$. Formalmente, el modelo de la empresa Accel es el siguiente:

$$
\begin{gathered}
\hat{p}_{t}=1.000212 p_{t-1}+\varepsilon_{t}, \\
\sigma_{t}^{2}=4.83 \times 10^{-5}+.149125 \varepsilon_{t-1}^{2}-0.051758 \varepsilon_{t-2}^{2}+0.855456 \sigma_{t-1}^{2} .
\end{gathered}
$$

Este modelo servirá para hacer los pronósticos ex ante de los precios en los siguientes lapsos: 1 año; 6 meses, 3 meses y un mes; 15 días y una semana. Recordemos que lo que se pretende es obtener los errores del pronóstico, $\varepsilon_{t}$, más pequeños posibles.

\begin{tabular}{|c|c|c|}
\hline Empresa & \multicolumn{2}{|c|}{ Modelo } \\
\hline \multirow{7}{*}{ Accel } & Variable & Coefficient \\
\hline & $\operatorname{AR}(1)$ & 1.000212 \\
\hline & \multicolumn{2}{|c|}{ Variance Equation } \\
\hline & $\mathrm{C}$ & $4.83 \times 10^{-5}$ \\
\hline & $\operatorname{ARCH}(1)$ & 0.149125 \\
\hline & $\mathrm{ARCH}(2)$ & -0.051758 \\
\hline & GARCH(1) & 0.855456 \\
\hline \multirow{3}{*}{ Gpo. Palacio de $\mathrm{H}$. } & Variable & Coefficient \\
\hline & $\mathrm{AR}(1)$ & 0.999908 \\
\hline & $\mathrm{MA}(1)$ & 0.053795 \\
\hline \multirow{11}{*}{ Cemex } & Variable & Coefficient \\
\hline & $\operatorname{AR}(1)$ & 0.543155 \\
\hline & $\mathrm{AR}(2)$ & 0.399808 \\
\hline & $\mathrm{MA}(1)$ & 0.761626 \\
\hline & $\mathrm{MA}(6)$ & 0.340318 \\
\hline & \multicolumn{2}{|c|}{ Variance Equation } \\
\hline & $\mathrm{C}$ & 5.506929 \\
\hline & $\mathrm{ARCH}(1)$ & -1.376097 \\
\hline & $\mathrm{ARCH}(2)$ & -1.217323 \\
\hline & GARCH(1) & -0.736923 \\
\hline & $\operatorname{GARCH}(2)$ & -0.805591 \\
\hline
\end{tabular}

Tabla 3. Correcciones econométricas. 
Tabla 3. Gorrecciones econométricas (continuación).

\begin{tabular}{|c|c|c|}
\hline \multirow{8}{*}{ Gpo. Continental } & Variable & Coefficient \\
\hline & $\mathrm{AR}(1)$ & 0.977649 \\
\hline & $\mathrm{MA}(2)$ & 0.564433 \\
\hline & $\mathrm{MA}(7)$ & 0.331659 \\
\hline & \multicolumn{2}{|c|}{ Variance Equation } \\
\hline & $\mathrm{C}$ & 4.908246 \\
\hline & $\mathrm{ARCH}(1)$ & -0.413126 \\
\hline & $\operatorname{GARCH}(1)$ & -0.804784 \\
\hline \multirow{9}{*}{ Cydsa } & Variable & Coefficient \\
\hline & $\mathrm{AR}(1)$ & 0.662204 \\
\hline & $\mathrm{AR}(3)$ & 0.399248 \\
\hline & $\mathrm{MA}(1)$ & 0.699983 \\
\hline & $\mathrm{MA}(3)$ & -0.253240 \\
\hline & \multicolumn{2}{|c|}{ Variance Equation } \\
\hline & $\mathrm{C}$ & 86.93235 \\
\hline & $\mathrm{ARCH}(1)$ & -2.249711 \\
\hline & GARCH(1) & -0.996035 \\
\hline \multirow{8}{*}{ Ekco } & Variable & Coefficient \\
\hline & $\operatorname{AR}(1)$ & 1.037600 \\
\hline & $\operatorname{AR}(8)$ & -0.038633 \\
\hline & $\mathrm{MA}(7)$ & -0.095639 \\
\hline & \multicolumn{2}{|c|}{ Variance Equation } \\
\hline & $\mathrm{C}$ & 0.000117 \\
\hline & $\operatorname{ARCH}(1)$ & 0.146214 \\
\hline & GARCH(1) & 0.587726 \\
\hline \multirow{4}{*}{ Gpo. S \& Rocha } & Variable & Coefficient \\
\hline & $\operatorname{AR}(1)$ & 0.998963 \\
\hline & $\mathrm{MA}(1)$ & 0.088713 \\
\hline & $\overline{\mathrm{MA}(2)}$ & 0.057343 \\
\hline \multirow{11}{*}{ GF Santander } & Variable & Coefficient \\
\hline & $\operatorname{AR}(1)$ & 0.740971 \\
\hline & $\mathrm{AR}(2)$ & 0.274002 \\
\hline & $\mathrm{MA}(1)$ & 0.821013 \\
\hline & $\mathrm{MA}(2)$ & 0.732451 \\
\hline & \multicolumn{2}{|c|}{ Variance Equation } \\
\hline & $\mathrm{C}$ & 695.6106 \\
\hline & $\mathrm{ARCH}(1)$ & -3.649813 \\
\hline & $\mathrm{ARCH}(2)$ & -2.012759 \\
\hline & $\operatorname{GARCH}(1)$ & -1.000051 \\
\hline & $\operatorname{GARCH}(2)$ & -0.984493 \\
\hline
\end{tabular}


Tabla 3. Correcciones econométricas (continuación).

\begin{tabular}{|c|c|c|}
\hline \multirow{8}{*}{ San Luis Corp. } & Variable & Coefficient \\
\hline & $\operatorname{AR}(1)$ & 0.993284 \\
\hline & $\mathrm{MA}(1)$ & 0.089571 \\
\hline & $\mathrm{MA}(2)$ & 0.069860 \\
\hline & & \\
\hline & $\mathrm{C}$ & $1.04 \times 10^{-6}$ \\
\hline & $\overline{\mathrm{ARCH}}(1)$ & 0.091638 \\
\hline & GARCH(1) & 0.939601 \\
\hline \multirow{11}{*}{ Sidek } & Variable & Coefficient \\
\hline & $\overline{\operatorname{AR}}(1)$ & 0.997040 \\
\hline & $\mathrm{MA}(1)$ & 0.409629 \\
\hline & $\mathrm{MA}(9)$ & -0.039580 \\
\hline & $\mathrm{MA}(13)$ & -0.024640 \\
\hline & $\mathrm{MA}(6)$ & 0.009417 \\
\hline & & \\
\hline & $\mathrm{C}$ & 0.026218 \\
\hline & $\operatorname{ARCH}(1)$ & 0.895195 \\
\hline & $\mathrm{ARCH}(2)$ & 0.873803 \\
\hline & GARCH(1) & -0.983462 \\
\hline \multirow{8}{*}{ Situr } & Variable & Coefficient \\
\hline & $\overline{\mathrm{AR}}(1)$ & $0 . \overline{949207}$ \\
\hline & $\mathrm{MA}(1)$ & 0.869469 \\
\hline & & \\
\hline & $\mathrm{C}$ & 0.456989 \\
\hline & $\overline{A R C H}(1)$ & 0.237403 \\
\hline & $\mathrm{ARCH}(2)$ & 0.336425 \\
\hline & GARCH(1) & -0.752920 \\
\hline \multirow{7}{*}{ Alfa } & Variable & Coefficient \\
\hline & $\mathrm{AR}(1)$ & 0.972810 \\
\hline & $\mathrm{MA}(1)$ & 0.856702 \\
\hline & & \\
\hline & $\mathrm{C}$ & 454.7555 \\
\hline & $\operatorname{ARCH}(1)$ & -1.857118 \\
\hline & GARCH(1) & -0.997736 \\
\hline \multirow{7}{*}{ Banamex } & Variable & Coefficient \\
\hline & $\operatorname{AR}(2)$ & 0.808887 \\
\hline & $\operatorname{AR}(6)$ & 0.171605 \\
\hline & \multicolumn{2}{|c|}{ Variance Equation } \\
\hline & $\mathrm{C}$ & 61.37083 \\
\hline & $\overline{\mathrm{ARCH}(1)}$ & -0.643373 \\
\hline & GARCH(1) & -0.999180 \\
\hline
\end{tabular}


Tabla 3. Correcciones econométricas (continuación).

\begin{tabular}{|c|c|c|}
\hline \multirow{8}{*}{ DESC } & Variable & Coefficient \\
\hline & $\operatorname{AR}(1)$ & 1.000994 \\
\hline & $\mathrm{MA}(1)$ & 0.177991 \\
\hline & \multicolumn{2}{|c|}{ Variance Equation } \\
\hline & $\mathrm{C}$ & $2.42 \times 10^{-5}$ \\
\hline & $\operatorname{ARCH}(1)$ & 0.121597 \\
\hline & GARCH(1) & 0.416458 \\
\hline & GARCH $(2)$ & 0.475301 \\
\hline \multirow{7}{*}{ FEMSA } & Variable & Coefficient \\
\hline & $\operatorname{AR}(1)$ & 1.017738 \\
\hline & $\mathrm{MA}(2)$ & 0.467244 \\
\hline & \multicolumn{2}{|c|}{ Variance Equation } \\
\hline & $\mathrm{C}$ & 58.58106 \\
\hline & $\mathrm{ARCH}(1)$ & -0.552006 \\
\hline & GARCH(1) & -0.999810 \\
\hline \multirow{8}{*}{ Gpo. Carso } & Variable & Coefficient \\
\hline & $\operatorname{AR}(1)$ & 1.027686 \\
\hline & $\mathrm{MA}(1)$ & 0.175859 \\
\hline & $\mathrm{MA}(10)$ & 0.521070 \\
\hline & \multicolumn{2}{|c|}{ Variance Equation } \\
\hline & $\mathrm{C}$ & 849.4672 \\
\hline & $\mathrm{ARCH}(1)$ & -1.263032 \\
\hline & GARCH(1) & -0.999904 \\
\hline \multirow{9}{*}{ Gpo. Ind. Saltillo } & Variable & Coefficient \\
\hline & $\operatorname{AR}(1)$ & 0.903097 \\
\hline & $\operatorname{AR}(7)$ & 0.097340 \\
\hline & $\operatorname{MA}(7)$ & 0.325922 \\
\hline & $\mathrm{MA}(10)$ & 0.520576 \\
\hline & \multicolumn{2}{|c|}{ Variance Equation } \\
\hline & $\mathrm{C}$ & 76.06649 \\
\hline & $\mathrm{ARCH}(1)$ & -1.534949 \\
\hline & GARCH(1) & -0.999899 \\
\hline \multirow{11}{*}{ Peñoles Ind. } & Variable & Coefficient \\
\hline & $\operatorname{AR}(1)$ & 0.757309 \\
\hline & $\operatorname{AR}(5)$ & 0.328449 \\
\hline & $\mathrm{MA}(2)$ & 0.695712 \\
\hline & $\mathrm{MA}(7)$ & 0.337317 \\
\hline & \multicolumn{2}{|c|}{ Variance Equation } \\
\hline & $\mathrm{C}$ & 265.1441 \\
\hline & $\mathrm{ARCH}(1)$ & -2.174714 \\
\hline & $\mathrm{ARCH}(2)$ & -3.990563 \\
\hline & GARCH(1) & -1.000031 \\
\hline & GARCH(2) & -0.950552 \\
\hline
\end{tabular}


Tabla 3. Correcciones econométricas (continuación).

\begin{tabular}{|c|c|c|}
\hline \multirow{9}{*}{ Savia } & Variable & Coefficient \\
\hline & $\operatorname{AR}(1)$ & 0.692628 \\
\hline & $\operatorname{AR}(2)$ & 0.252381 \\
\hline & $\overline{\mathrm{MA}(1)}$ & 1.518754 \\
\hline & MA $(2)$ & 0.953879 \\
\hline & & \\
\hline & $\mathrm{C}$ & 725.6042 \\
\hline & $\operatorname{ARCH}(1)$ & -5.643734 \\
\hline & GARCH(1) & -0.997976 \\
\hline \multirow{10}{*}{ Telmex } & Variable & Coefficient \\
\hline & $\mathrm{AR}(1)$ & 0.721977 \\
\hline & $\mathrm{AR}(5)$ & 0.281215 \\
\hline & $\mathrm{MA}(5)$ & 0.671203 \\
\hline & & \\
\hline & $\mathrm{C}$ & 6.736029 \\
\hline & $\mathrm{ARCH}(1)$ & -0.395172 \\
\hline & $\mathrm{ARCH}(2)$ & -0.464484 \\
\hline & GARCH(1) & -0.999937 \\
\hline & GARCH $(2)$ & -0.998251 \\
\hline \multirow{8}{*}{ Televisa } & Variable & Coefficient \\
\hline & $\operatorname{AR}(1)$ & 1.000618 \\
\hline & $\mathrm{MA}(1)$ & 0.130020 \\
\hline & $\mathrm{MA}(10)$ & 0.048461 \\
\hline & $=$ & \\
\hline & $\mathrm{C}$ & $3.68 \times 10^{-6}$ \\
\hline & $\operatorname{ARCH}(1)$ & 0.023770 \\
\hline & GARCH(1) & 0.979253 \\
\hline \multirow{10}{*}{ Tubos de Acero } & Variable & Coefficient \\
\hline & $\operatorname{AR}(1)$ & 1.134596 \\
\hline & $\mathrm{MA}(1)$ & 0.641385 \\
\hline & $\mathrm{MA}(7)$ & 0.245898 \\
\hline & $\mathrm{MA}(4)$ & 0.322587 \\
\hline & $\mathrm{MA}(10)$ & 0.260037 \\
\hline & \multicolumn{2}{|c|}{ Variance Equation } \\
\hline & $\mathrm{C}$ & 3577.986 \\
\hline & $\mathrm{ARCH}(1)$ & -1.884279 \\
\hline & GARCH(1) & -0.998123 \\
\hline
\end{tabular}




\subsubsection{Resultados con un año de datos}

Las tablas con los resultados de la estimación AR(1) y la corrección de ARMAGARCH usando un año, seis y tres meses de datos se pueden consultar en el Apéndice 1. El primer resultado surge de la comparación entre la evaluación de eficiencia en forma débil usando toda la serie histórica y usando sólo un año de datos. En este caso, la ineficiencia tiende a desaparecer conforme se reduce el lapso de tiempo analizado: usando toda la serie histórica se presentaron 22 casos de ineficiencia ( $70.96 \%$ de la muestra), usando sólo un año de datos se presentaron sólo 9 ( $29 \%$ de la muestra). Segundo, usando un año de datos sólo ocho casos resultaron heteroscedásticos, comparado con 28 cuando se emplea toda la serie histórica de los precios de cierre diarios de cada acción.

\subsubsection{Resultados con seis meses de datos}

Sólo cuatro casos de ineficiencia, de los cuales sólo uno pertenece a la muestra del IPC.

\subsubsection{Resultados con tres meses de datos}

De la observación de los apartados anteriores, es factible aseverar lo siguiente:

i) Las ineficiencias y heteroscedasticidades que se observan cuando se usa toda la serie de datos ( 8 años) tienden a desaparecer conforme se reduce la longitud de las series de tiempo modeladas, de tal forma que cuando se usaron sólo tres meses de datos, únicamente se observaron 3 casos de ineficiencia.

ii) Asimismo, la corrección ARMA-GARCH de las series de tiempo ineficientes se vuelve más sencilla.

iii) Un problema de usar series de tiempo demasiado cortas es que cuando las empresas son poco bursátiles, sus series pueden resultar constantes. Los casos de Patria Reasegurador serie B y Pepsigx serie B son algunos ejemplos.

iv) De acuerdo con la aseveración de Loughran y Ritter (2000), es más probable observar ineficiencias en las empresas que no están muestreadas en los portafolios de mercado (IPC en este caso).

\subsubsection{El poder predictivo de los modelos estimados}

En este apartado se prueba el poder predictivo ex ante de los modelos estimados en las secciones anteriores. Los resultados de los pronósticos se presentan para seis diferentes casos: un año, 6 meses, 3 meses, un mes, 15 días y una semana. Los modelos estimados usan diferentes longuitudes de la serie de datos: 8 años, un año, 6 meses y 3 meses. Además, como ya se mencionó, la elección de los modelos con el mayor poder predictivo ex ante dependerá del error de pronóstico medio, así como de la longitud del intervalo generado al $90 \%$ de confianza estadística. La consideración de este último punto resulta ser importante ya que el cálculo de un pronóstico puntual es imposible; por tal motivo, el margen de error de nuestros pronósticos está dado precisamente por este intervalo. Por ejemplo, para el caso de un año de pronósticos usando los modelos estimados con 6 meses de datos, tenemos que el error medio de pronóstico es de $\$ 3.72$ pesos. Sin embargo, el margen de error que podemos cometer es hasta en $\$ 12.75$ pesos, el cual es el límite superior del intervalo. ${ }^{1}$

1 El caso negativo (-\$2.65, el límite inferior del intervalo) no tiene sentido económico, pero implicaría el hecho de que las empresas pueden quebrar. 
Tabla 4. Datos de errores de pronóstico.

A un año (sin Patria ni Pepsigx).

\begin{tabular}{|c|c|c|c|c|}
\hline & Toda la serie & Un año & 6 meses & 3 meses \\
\hline Media & $-2.6 \times 10^{13}$ & 2.726739 & 3.724139 & 6.966095 \\
\hline Desviación estándar & $1.04 \times 10^{14}$ & 3.835371 & 3.888066 & 4.192202 \\
\hline Límite inferior & $-2 \times 10^{13}$ & -3.56327 & -2.65229 & 0.090883 \\
\hline Límite superior & $1.44 \times 10^{13}$ & 9.016748 & 10.10057 & 13.84131 \\
\hline Longitud del Intervalo & $3.44 \times 10^{13}$ & 12.580018 & 12.75286 & 13.932193 \\
\hline
\end{tabular}

A seis meses ( $\sin$ Patria ni Pepsigx).

\begin{tabular}{|c|c|c|c|c|}
\hline & Toda la serie & Un año & 6 meses & 3 meses \\
\hline Media & -2708876 & 3.271149 & 3.652198 & 5.495878 \\
\hline Desviación estándar & 7263458 & 2.907041 & 3.01096 & 3.554443 \\
\hline Límite inferior & $-1.5 \times 10^{7}$ & -1.4964 & -1.28578 & -0.33341 \\
\hline Límite superior & 9203195 & 8.038697 & 8.590172 & 11.32516 \\
\hline Longitud del intervalo & $2.4 \times 10^{7}$ & 9.535097 & 9.875952 & 11.65857 \\
\hline
\end{tabular}

A tres meses (sin Patria ni Pepsigx).

\begin{tabular}{|c|c|c|c|c|}
\hline & Toda la serie & Un año & 6 meses & 3 meses \\
\hline Media & -1661.77 & 2.108322 & 2.147511 & 3.205773 \\
\hline Desviación estándar & 2957.424 & 2.088337 & 2.232896 & 2.58289 \\
\hline Límite inferior & -6511.95 & -1.31655 & -1.51444 & -1.03017 \\
\hline Límite superior & 3188.403 & 5.533195 & 5.80946 & 7.441714 \\
\hline Longitud del intervalo & 9700.353 & 6.849745 & 7.3239 & 8.471884 \\
\hline
\end{tabular}


Tabla 4. Datos de errores de pronóstico (continuación).

A un mes (sin Patria ni Pepsigx).

\begin{tabular}{|c|c|c|c|c|}
\hline & Toda la serie & Un año & 6 meses & 3 meses \\
\hline Media & -14.7195 & 0.828873 & 0.632848 & 1.048886 \\
\hline Desviación estándar & 15.59599 & 0.943141 & 0.951828 & 1.058633 \\
\hline Límite inferior & -40.297 & -0.71788 & -0.92815 & -0.68727 \\
\hline Límite superior & 10.8579 & 2.375624 & 2.193845 & 2.785045 \\
\hline Longitud del intervalo & 51.1549 & 3.093504 & 3.121995 & 3.472315 \\
\hline
\end{tabular}

A 15 días (sin Patria ni Pepsigx).

\begin{tabular}{|c|c|c|c|c|}
\hline & Toda la serie & Un año & 6 meses & 3 meses \\
\hline Media & -3.81873 & 0.427828 & 0.20653 & 0.428565 \\
\hline Desviación estándar & 3.917657 & 0.665349 & 0.612282 & 0.656159 \\
\hline Límite inferior & -10.2437 & -0.66335 & -0.79761 & -0.64754 \\
\hline Límite superior & 2.60623 & 1.519001 & 1.210673 & 1.504666 \\
\hline Longitud del intervalo & 12.84993 & 2.182351 & 2.008283 & 2.152206 \\
\hline
\end{tabular}

A 1 semana (sin Patria ni Pepsigx).

\begin{tabular}{|c|c|c|c|c|}
\hline & Toda la serie & Un año & 6 meses & 3 meses \\
\hline Media & -1.42399 & 0.477563 & 0.285673 & 0.411053 \\
\hline Desviación estándar & 1.546 & 0.625178 & 0.55341 & 0.589834 \\
\hline Límite inferior & -3.95943 & -0.54773 & -0.62192 & -0.55627 \\
\hline Límite superior & 1.111451 & 1.502855 & 1.193266 & 1.37838 \\
\hline Longitud del intervalo & 5.070881 & 2.050585 & 1.815186 & 1.93465 \\
\hline
\end{tabular}


Conviene mencionar que cuando se estimaron los modelos con tres meses de datos, los casos de Patria Reasegurador serie B y Pepsigx serie B resultaron constantes. Por ello, y para que los resultados fueran comparables, se eliminaron de la muestra. Como se puede observar, cuando se pronostican precios desde tres meses hasta un año, los modelos estimados usando un año de datos producen los mejores resultados, en otras palabras, obtenemos los errores medios de pronósticos más bajos y los intervalos más cortos.

Para pronosticar precios menores a un mes, conviene usar 6 meses de datos. El caso de un mes de pronósticos es sui generis. Finalmente hay que mencionar que los modelos estimados usando 6 meses de datos producen el error medio de pronóstico más pequeño; pero el intervalo generado por los modelos estimados con un año de datos es el más corto. En estos casos, y por los motivos explicados anteriormente, se opta por el modelo que genera los intervalos más cortos.

\section{Conclusiones}

Como se mencionó en la introducción, los objetivos de este trabajo son: primero, determinar una metodología para la construcción de modelos econométricos con un alto poder predictivo ex ante. Una consecuencia inmediata de este primer objetivo fue el análisis de la eficiencia en forma débil de los precios de cierre diarios de una muestra de 31 empresas, que incluyó empresas muestreadas en el IPC y empresas que no lo están. Segundo, determinar los modelos con el mayor poder predictivo ex ante. Para lo anterior se usó la modelación ARMA-GARCH y se tomaron series de tiempo de diferente longitud. Se excluyó el último año de datos de cada serie de tiempo accionaria para poder probar el poder predictivo ex ante de cada modelo, variando la longitud de los pronósticos. En otras palabras, se realizaron pronósticos a un año, a seis meses, a tres meses y a un mes, así como a 15 días y a una semana.

Los resultados principales son tres: primero, la metodología para la construcción de modelos de pronóstico de precios accionarios se resume en la Figura 1, y se describe de la siguiente manera: $i$ ) Correr las series de tiempo accionarias como modelos autorregresivos de primer orden, $\mathrm{AR}(1)$. Si el modelo resulta ser no correlacionado serialmente y es homoscedástico, podemos concluir que la serie es eficiente en forma débil y podemos construir los pronósticos y ii) Si el modelo no cumple con estas dos condiciones mencionadas arriba, entonces se dice ineficiente y se corrige mediante la modelación ARMA-GARCH.

En torno a esta primera observación econométrica, las series accionarias de la Bolsa Mexicana de Valores muestran ineficiencia y heteroscedasticidad, la cual tiende a desaparecer conforme se reduce la longitud de las series. Cuando se usaron series de ocho años, 1991-1999, 70.96\% de las empresas muestreadas resultaron ineficientes y $90.32 \%$ heteroscedásticas; en comparación con $9.67 \%$ ineficientes y $16.12 \%$ heteroscedásticas cuando se utilizaron series de tiempo de 3 meses de longitud. Este hecho puede explicar por qué Moctezuma (1998) encontró ineficiencia en el IPC. La muestra de los rendimientos diarios que él emplea para realizar su análisis consistió precisamente de 8 años de datos. 
Figura 1. Diagrama de flujo para la modelación de series de tiempo.

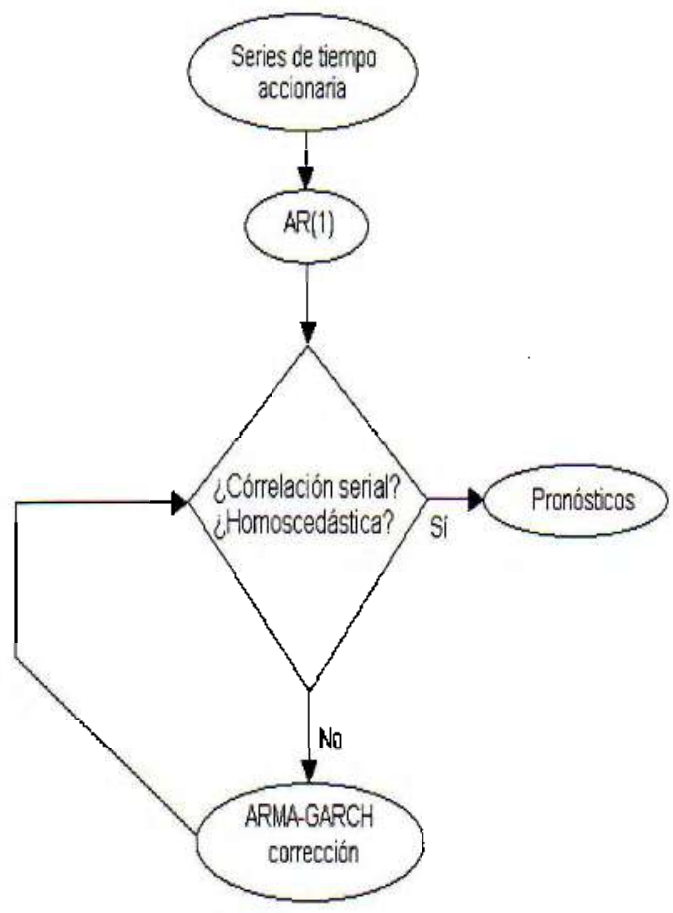

En respuesta al segundo objetivo planteado, y quizá el resultado más importante, es que se encontró que los modelos estimados tomando series de un año de longitud producen los mejores pronósticos a un año, seis, tres y un mes. Mientras que los modelos con una serie de datos de 6 meses producen mejores resultados a 15 días y una semana (el error medio de pronóstico a una semana fue de $\$ 0.28$ y la longitud del intervalo al $90 \%$ de confianza estadística de $\$ 1.81$ ). Esto permite afirmar que aunque la eficiencia en forma débil merma el poder predictivo ex ante de los modelos ARMA-GARCH, en el sentido de que impide el pronóstico puntual, aún nos permite hacer pronósticos bastante acertados.

Por último, algunos resultados secundarios: i) Como se puede observar, el mercado accionario mexicano es eficiente en general y $i$ i) Es más factible hallar ineficiencias fuera de las muestras de los portafolios de mercado, el IPC en el caso de México. 
A1. Un año de datos.

\begin{tabular}{|c|c|c|c|c|c|c|}
\hline Empresa & $\bar{R}^{2}$ & Durbin-Watson & $\begin{array}{c}\text { Probabilidad } \\
\text { Breusch-Godfrey } \\
\text { correlación serial }\end{array}$ & $\begin{array}{c}\text { Coef. } \\
\alpha_{1}\end{array}$ & $\begin{array}{c}\text { Probabilidad } \\
\text { ARCH-LM }\end{array}$ & ¿Eficiencia? \\
\hline Accel & 0.937111 & 2.305383 & 0.042686 & 1.000334 & 0.824580 & No \\
\hline Palacio de Hierro & 0.967386 & $1 . \overline{717858}$ & 0.011097 & 0.999318 & 0.540693 & $\overline{\mathrm{No}}$ \\
\hline Cemex & 0.980830 & 1.809026 & 0.313413 & 0.998246 & 0.963113 & $\overline{\text { Sí }}$ \\
\hline Gpo. Continental & 0.966718 & 2.115443 & 0.296912 & 0.999436 & 0.051624 & Sí \\
\hline Cydsa & 0.983174 & 1.608935 & 0.002326 & 1.000363 & 0.000000 & No \\
\hline Ekco & 0.976111 & 1.992465 & 0.974562 & 0.997217 & $0 . \overline{811047}$ & Sí \\
\hline Gpo. S \& Rocha & 0.970654 & 1.796175 & 0.227503 & $0 . \overline{991639}$ & 0.979192 & Sí \\
\hline GF Santander & 0.993033 & 1.722600 & 0.084503 & 1.001475 & 0.976828 & Sí \\
\hline \multicolumn{7}{|l|}{ Patria } \\
\hline Pepsigx & 0.994648 & 1.005068 & 0.000000 & 0.999298 & 0.000000 & $\overline{\mathrm{No}}$ \\
\hline San Luis Corp. & 0.822208 & 2.092722 & 0.000028 & 1.000091 & 0.000035 & $\overline{\mathrm{No}}$ \\
\hline Sidek & 0.965125 & 1.585263 & 0.000114 & 0.996112 & 0.104294 & No \\
\hline Situr & 0.960872 & 1.955251 & 0.945968 & 0.996926 & 0.000035 & Sí \\
\hline TMM & 0.973735 & 1.774268 & 0.219226 & $0.9 \overline{98785}$ & $0.74767 \overline{7}$ & Sí \\
\hline Alfa & 0.962481 & 1.574815 & 0.000018 & 1.000798 & 0.000000 & $\mathrm{No}$ \\
\hline Apasco & 0.987862 & 1.798648 & 0.344399 & 1.001128 & 0.682942 & Sí \\
\hline Banamex & 0.982795 & 1.770393 & 0.081043 & 1.000496 & 0.862682 & Sí \\
\hline Bimbo & 0.928770 & 1.998165 & 0.947429 & 1.000222 & 0.789007 & $\overline{\text { Sí }}$ \\
\hline $\mathrm{DESC}$ & 0.959150 & 1.953705 & 0.942329 & 0.999969 & 0.314165 & Sí \\
\hline FEMSA & 0.976163 & $1.72 \overline{28526}$ & 0.046011 & 1.000591 & 0.476249 & Sí \\
\hline
\end{tabular}


A1. Un año de datos (continuación).

\begin{tabular}{|c|c|c|c|c|c|c|}
\hline Empresa & $\bar{R}^{2}$ & Durbin-Watson & $\begin{array}{c}\text { Probabilidad } \\
\text { Breusch-Godfrey } \\
\text { correlación serial }\end{array}$ & $\begin{array}{c}\text { Coef. } \\
\alpha_{1}\end{array}$ & $\begin{array}{c}\text { Probabilidad } \\
\text { ARCH-LM }\end{array}$ & ¿Eficiencia? \\
\hline Gpo. Carso & 0.961918 & 1.932360 & 0.874238 & 1.000320 & 0.492079 & Sí \\
\hline Gpo. Ind. Saltillo & 0.986438 & 1.667154 & 0.033693 & 1.001155 & 0.008214 & No \\
\hline Kimberly Clark & 0.968472 & 1.871302 & 0.614942 & 1.000332 & 0.970944 & Sí \\
\hline Peñoles Ind. & 0.903474 & 1.856976 & 0.132834 & 0.999764 & 0.030367 & Sí \\
\hline Savia & 0.970898 & 1.906053 & 0.702354 & 0.999258 & 0.365229 & Sí \\
\hline Soriana & 0.971842 & 1.953626 & 0.360803 & 1.000896 & 0.024169 & Sí \\
\hline Telmex & 0.986006 & 1.861658 & 0.485134 & 1.001298 & 0.793691 & Sí \\
\hline Televisa & 0.983089 & 1.907771 & 0.614910 & 1.000890 & 0.601411 & Sí \\
\hline Tubos de Acero & 0.967775 & 1.663120 & 0.020738 & 1.001558 & 0.257000 & No \\
\hline Vitro & 0.949287 & 1.984349 & 0.898017 & 0.999523 & 0.272246 & Sí \\
\hline Walmart & 0.974117 & 2.052694 & 0.599807 & 1.000143 & 0.140851 & Sí \\
\hline
\end{tabular}


A1. Un año de datos (continuación).

\begin{tabular}{|c|c|c|}
\hline Empresa & \multicolumn{2}{|c|}{ Modelo } \\
\hline \multirow{4}{*}{ Accel } & Variable & Coefficient \\
\hline & $\mathrm{AR}(1)$ & 1.000454 \\
\hline & $\overline{\mathrm{MA}}(1)$ & -0.189837 \\
\hline & $\mathrm{MA}(8)$ & -0.149852 \\
\hline \multirow{5}{*}{ Gpo. Palacio de Hierro } & Variable & Coefficient \\
\hline & $\operatorname{AR}(1)$ & 0.999152 \\
\hline & $\mathrm{MA}(1)$ & 0.128976 \\
\hline & $\mathrm{MA}(2)$ & 0.154790 \\
\hline & $\mathrm{MA}(5)$ & 0.182822 \\
\hline \multirow{8}{*}{ Cydsa } & Variable & Coefficient \\
\hline & $\operatorname{AR}(1)$ & 0.702561 \\
\hline & $\mathrm{MA}(1)$ & 1.355103 \\
\hline & $\mathrm{MA}(2)$ & 0.955128 \\
\hline & \multicolumn{2}{|c|}{ Variance Equation } \\
\hline & $\mathrm{C}$ & 26.01917 \\
\hline & $\overline{\mathrm{ARCH}}(1)$ & -2.345098 \\
\hline & GARCH(1) & -0.894687 \\
\hline \multirow{6}{*}{ Pepsigx } & Variable & Coefficient \\
\hline & $\operatorname{AR}(1)$ & 1.041369 \\
\hline & \multicolumn{2}{|c|}{ Variance Equation } \\
\hline & $\mathrm{C}$ & 1.102911 \\
\hline & $\overline{\mathrm{ARCH}(1)}$ & -2.553190 \\
\hline & $\mathrm{GARCH}(1)$ & -0.728990 \\
\hline \multirow{5}{*}{ San Luis Corp. } & Variable & Coefficient \\
\hline & $\mathrm{AR}(1)$ & 1.144536 \\
\hline & $\operatorname{AR}(3)$ & -0.144324 \\
\hline & $\mathrm{MA}(1)$ & -0.321276 \\
\hline & $\mathrm{MA}(2)$ & -0.448135 \\
\hline
\end{tabular}


A1. Un año de datos (continuación).

\begin{tabular}{|c|c|c|}
\hline \multirow{3}{*}{ Sidek } & Variable & Coefficient \\
\hline & $\mathrm{AR}(1)$ & 0.995395 \\
\hline & $\mathrm{MA}(2)$ & 0.199256 \\
\hline \multirow{6}{*}{ Situr } & Variable & Coefficient \\
\hline & $\mathrm{AR}(1)$ & 0.991858 \\
\hline & \multicolumn{2}{|c|}{ Variance Equation } \\
\hline & $\mathrm{C}$ & $8.62 \times 10^{-5}$ \\
\hline & $\operatorname{ARCH}(1)$ & 0.289711 \\
\hline & $\mathrm{GARCH}(1)$ & 0.361721 \\
\hline \multirow{3}{*}{ Alfa } & Variable & Coefficient \\
\hline & $\operatorname{AR}(1)$ & 1.000526 \\
\hline & $\mathrm{MA}(1)$ & 0.285561 \\
\hline \multirow{3}{*}{ Gpo. Ind. Saltillo } & Variable & Coefficient \\
\hline & $\mathrm{AR}(1)$ & 1.001057 \\
\hline & $\mathrm{MA}(1)$ & 0.163631 \\
\hline \multirow{3}{*}{ Peñoles Ind. } & Variable & Coefficient \\
\hline & $\mathrm{AR}(1)$ & 0.999870 \\
\hline & $\mathrm{MA}(4)$ & -0.169980 \\
\hline \multirow{7}{*}{ Soriana } & Variable & Coefficient \\
\hline & $\mathrm{AR}(1)$ & 0.999819 \\
\hline & $\mathrm{MA}(4)$ & -0.736966 \\
\hline & \multicolumn{2}{|c|}{ Variance Equation } \\
\hline & $\mathrm{C}$ & 749.9158 \\
\hline & $\operatorname{ARCH}(1)$ & -1.074215 \\
\hline & $\mathrm{GARCH}(1)$ & -0.999659 \\
\hline \multirow{3}{*}{ Tubos de Acero } & Variable & Coefficient \\
\hline & $\operatorname{AR}(1)$ & 1.001398 \\
\hline & $\mathrm{MA}(1)$ & 0.183383 \\
\hline
\end{tabular}


A2. Seis meses de datos.

\begin{tabular}{|c|c|c|c|c|c|c|}
\hline Empresa & $\bar{R}^{2}$ & Durbin-Watson & $\begin{array}{c}\text { Probabilidad } \\
\text { Breusch-Godfrey } \\
\text { correlación serial }\end{array}$ & $\begin{array}{c}\text { Coef. } \\
\alpha_{1}\end{array}$ & $\begin{array}{c}\text { Probabilidad } \\
\text { ARCH-LM }\end{array}$ & ¿Eficiencia? \\
\hline Accel & 0.765732 & 2.746474 & 0.000047 & 0.999139 & 0.003943 & No \\
\hline Palacio de Hierro & 0.983003 & 1.943775 & 0.718265 & 0.996632 & 0.780252 & Sí \\
\hline Cemex & 0.972503 & 1.883143 & 0.801045 & $0 . \overline{994244}$ & 0.891215 & $\overline{\text { Sí }}$ \\
\hline Gpo. Continental & 0.970471 & 2.182657 & 0.271550 & 0.997464 & 0.807126 & Sí \\
\hline Cydsa & 0.912455 & 1.382906 & 0.000559 & 0.999849 & 0.000000 & No \\
\hline Ekco & 0.922218 & 1.966641 & 0.974898 & 0.997779 & 0.676490 & Sí \\
\hline Gpo. S\& Rocha & 0.982955 & 1.463363 & 0.000000 & 0.991197 & 0.091344 & No \\
\hline GF Santander & 0.958992 & 1.686660 & $0 . \overline{207972}$ & 1.001246 & 0.927679 & Sí \\
\hline \multicolumn{7}{|l|}{ Patria } \\
\hline Pepsigx & 0.915236 & 2.015186 & 0.992424 & 1.000121 & 0.930234 & $\overline{\text { Sí }}$ \\
\hline San Luis Corp. & 0.938283 & 2.004764 & 0.999259 & 1.000397 & 0.713250 & Sí \\
\hline Sidek & 0.962590 & 2.015394 & 0.758133 & 0.994208 & 0.860929 & Sí \\
\hline Situr & 0.973155 & 2.238206 & 0.204776 & 0.992473 & 0.560655 & Sí \\
\hline TMM & 0.845559 & 2.066307 & 0.914970 & 0.995918 & 0.886554 & Sí \\
\hline Alfa & 0.853989 & 1.620654 & 0.096344 & 0.999913 & 0.082703 & Sí \\
\hline Apasco & 0.893161 & 1.766237 & 0.466424 & 1.000440 & 0.952610 & Sí \\
\hline Banamex & 0.900399 & 1.645225 & 0.067901 & 0.999430 & 0.451544 & Sí \\
\hline Bimbo & 0.757773 & 2.025879 & 0.084779 & 0.999270 & 0.531785 & Sí \\
\hline DESC & 0.939987 & 1.594864 & 0.075299 & 0.998953 & 0.803917 & Sí \\
\hline FEMSA & 0.932296 & 1.628458 & 0.125487 & 1.000105 & 0.384702 & $\overline{\text { Sí }}$ \\
\hline
\end{tabular}


A2. Seis meses de datos (continuación).

\begin{tabular}{|c|c|c|c|c|c|c|}
\hline Empresa & $\bar{R}^{2}$ & Durbin-Watson & $\begin{array}{c}\text { Probabilidad } \\
\text { Breusch-Godfrey } \\
\text { correlación serial }\end{array}$ & $\begin{array}{c}\text { Coef. } \\
\alpha_{1}\end{array}$ & $\begin{array}{c}\text { Probabilidad } \\
\text { ARCH-LM }\end{array}$ & ¿Eficiencia? \\
\hline Gpo. Carso & 0.897353 & 2.055396 & 0.299795 & 0.999674 & 0.264929 & Sí \\
\hline Gpo. Ind. Saltillo & 0.929244 & 1.874000 & 0.863193 & 0.999767 & 0.691265 & Sí \\
\hline Kimberly Clark & 0.901775 & 2.013278 & 0.533431 & 0.999379 & 0.554441 & Sí \\
\hline Peñoles Ind. & 0.916001 & 1.763412 & 0.316544 & 0.998261 & 0.030156 & Sí \\
\hline Savia & 0.948056 & 1.766456 & 0.386444 & 0.999203 & 0.891464 & Sí \\
\hline Soriana & 0.908526 & 1.892563 & 0.464589 & 1.000350 & 0.058769 & Sí \\
\hline Telmex & 0.916775 & 1.745661 & 0.312051 & 1.000748 & 0.907737 & Sí \\
\hline Televisa & 0.942139 & 1.741300 & 0.250155 & 1.000419 & 0.860670 & \\
\hline Tubos de Acero & 0.917163 & 1.459548 & 0.000995 & 1.002030 & 0.426622 & No \\
\hline Vitro & 0.962187 & 1.636516 & 0.097589 & 0.999023 & $\mathbf{0 . 0 1 2 2 9 3}$ & Sí \\
\hline Walmart & $\mathbf{0 . 9 0 6 3 5 8}$ & 2.020089 & 0.782371 & 1.000180 & $\mathbf{0 . 0 4 4 3 5 5}$ & Sí \\
\hline
\end{tabular}


A2. Seis meses de datos (continuación).

\begin{tabular}{|c|c|c|}
\hline Empresa & \multicolumn{2}{|c|}{ Modelo } \\
\hline \multirow{3}{*}{ Accel } & Variable & Coefficient \\
\hline & $\mathrm{AR}(1)$ & 0.999271 \\
\hline & $\mathrm{MA}(1)$ & -0.409026 \\
\hline \multirow{3}{*}{ Cydsa } & Variable & Coefficient \\
\hline & $\mathrm{AR}(1)$ & 0.999723 \\
\hline & $\mathrm{MA}(1)$ & 0.336457 \\
\hline \multirow{4}{*}{ Gpo. S \& Rocha } & Variable & Coefficient \\
\hline & $\overline{A R}(1)$ & 0.989815 \\
\hline & $\mathrm{MA}(1)$ & 0.3551788 \\
\hline & $\mathrm{MA}(2)$ & 0.608797 \\
\hline \multirow{3}{*}{ Tubos de Acero } & Variable & Coefficient \\
\hline & $\mathrm{AR}(1)$ & 1.001788 \\
\hline & $\overline{\mathrm{MA}(1)}$ & 0.370158 \\
\hline
\end{tabular}


A3. Tres moses de datos.

\begin{tabular}{|c|c|c|c|c|c|c|}
\hline Empresa & $\bar{R}^{2}$ & Durbin-Watson & $\begin{array}{c}\text { Probabilidar } \\
\text { Breusch-Godfrey } \\
\text { corrclación serial }\end{array}$ & $\begin{array}{c}\text { Coef. } \\
\alpha_{1}\end{array}$ & $\begin{array}{c}\text { Probabilidad } \\
\text { ARCH-LM }\end{array}$ & ¿Eficiencia? \\
\hline Accel & 0.577522 & 2.871350 & 0.000488 & 0.999405 & 0.012129 & No \\
\hline Palacio de Hierro & -0.031210 & 1.999922 & 0.000116 & 0.999922 & 0.801782 & No \\
\hline Cemex & 0.867481 & 1.974329 & 0.948229 & 1.000176 & 0.615530 & Sí \\
\hline Gpo. Continental & 0.925575 & 1.965387 & 0.788284 & 0.997671 & 0.430526 & Sí \\
\hline Cydsa & 0.991020 & 1.486351 & 0.106290 & 0.997547 & 0.005828 & Sí \\
\hline Ekco & 0.850095 & 1.959839 & 0.693989 & 1.000512 & 0.002238 & Sí \\
\hline Gpo. S\& Rocha & 0.912336 & 1.780846 & 0.435931 & 0.940973 & 0.935012 & $\mathrm{Si}$ \\
\hline GF Santander & 0.945927 & 1.582794 & 0.234434 & 0.996835 & 0.797012 & Sí \\
\hline Patria & & & & & & $?$ \\
\hline Pepsigx & & & & 1 & & $?$ \\
\hline San Luis Corp. & 0.879039 & 2.000156 & 1.000000 & 0.999861 & 0.800865 & Sí \\
\hline Sidek & 0.978614 & 1.959181 & 0.931767 & 0.977601 & 0.284188 & Sí \\
\hline Situr & 0.966166 & 2.388272 & 0.280136 & 0.986261 & 0.576250 & Sí \\
\hline TMM & 0.801369 & 2.023354 & 0.990871 & 0.994838 & 0.900721 & Sí \\
\hline Alfa & 0.853249 & 1.483715 & 0.051095 & 1.001472 & 0.190640 & $\overline{\text { Sí }}$ \\
\hline Apasco & 0.898593 & 1.791184 & 0.750133 & 0.997956 & 0.484382 & Sí \\
\hline Banamex & 0.872046 & 1.488398 & 0.016020 & 0.996652 & 0.326874 & No \\
\hline Bimbo & 0.753137 & 1.936649 & 0.538419 & 1.000131 & 0.423901 & Sí \\
\hline DESC & 0.942486 & 1.550025 & 0.184978 & 0.997160 & 0.789128 & Sí \\
\hline FEMSA & 0.924312 & 1.572387 & 0.275950 & 0.997994 & 0.044655 & Sí \\
\hline Gpo. Carso & 0.891741 & 1.850109 & 0.060625 & 0.997243 & 0.341854 & Sí \\
\hline Gpo. Ind. Saltillo & 0.903948 & 1.847388 & 0.906147 & 0.998481 & 0.503698 & Sí \\
\hline
\end{tabular}


A3. Tres meses de datos (continuación).

\begin{tabular}{|c|c|c|c|c|c|c|}
\hline Empresa & $\bar{R}^{2}$ & Durbin-Watson & $\begin{array}{c}\text { Probabilidad } \\
\text { Breusch-Godfrey } \\
\text { correlación serial }\end{array}$ & $\begin{array}{c}\text { Coef. } \\
\alpha_{1}\end{array}$ & $\begin{array}{c}\text { Probabilidad } \\
\text { ARCH-LM }\end{array}$ & ¿Eficiencia? \\
\hline Kimberly Clark & 0.931603 & 1.845733 & 0.791171 & 0.997031 & 0.768383 & Sí \\
\hline Peñoles Ind. & 0.581392 & 2.066807 & 0.867846 & 1.000922 & 0.214034 & Sí \\
\hline Savia & 0.851444 & 2.189348 & 0.720797 & 0.999238 & 0.834999 & Sí \\
\hline Soriana & 0.780698 & 1.843656 & 0.648327 & 0.997778 & 0.007612 & Sí \\
\hline Telmex & 0.777536 & 1.764612 & 0.395936 & 0.999122 & 0.598829 & Sí \\
\hline Televisa & 0.929482 & 1.656613 & 0.397153 & 0.997263 & 0.454258 & Sí \\
\hline Tubos de Acero & 0.839884 & 1.568677 & 0.219253 & 1.000417 & 0.809978 & Sí \\
\hline Vitro & 0.956164 & 1.812435 & 0.603069 & 0.993302 & 0.655578 & Sí \\
\hline Walmart & 0.793122 & 2.156876 & 0.665662 & 0.997399 & 0.845272 & Sí \\
\hline
\end{tabular}

\begin{tabular}{|c|c|c|}
\hline Empresa & \multicolumn{2}{|c|}{ Modelo } \\
\hline \multirow{3}{*}{ Accel } & Variable & Coefficient \\
\hline & $\operatorname{AR}(1)$ & 0.999426 \\
\hline & $\mathrm{MA}(1)$ & 0.493935 \\
\hline \multirow{3}{*}{ Gpo. Palacio de Hierro } & Variable & Coefficient \\
\hline & $\mathrm{AR}(1)$ & 1.000114 \\
\hline & $\mathrm{MA}(2)$ & -0.979997 \\
\hline \multirow{3}{*}{ Banamex } & Variable & Coefficient \\
\hline & $\overline{\operatorname{AR}}(1)$ & $0 . \overline{995932}$ \\
\hline & $\overline{\mathrm{MA}(1)}$ & 0.395038 \\
\hline
\end{tabular}




\section{Bibliografía}

Bollerslev, T. (1986). Generalised Autoregressive Conditional Heteroskedasticity. Journal of Econometrics, 31, pp. 307-327.

Bollerslev, T. (1988). On the Correlation Structure for the Generalised Autoregressive Conditional Heteroskedasticity Process. Journal of Time Series Analysis, 9, pp. 121-132.

Chou, R. Y. (1988). Volatility Persistence and Stock Valuations: Some Empirical Evidence using GARCH. Journal of Applied Econometrics, 3, pp. 279-294.

Corhay, A., and A. T. Rad (1991). Conditional Heteroscedasticity in Stock Returns: international evidence. University of Limburg, Faculty of Economics Research. Memorandum 91-104.

Cowles, A. (1933). Can Stock Market Forecasters Forecast? Econometrica, 1, pp. 309-324.

Cowles, A., and H. E. Jones (1937). Some a posteriori Probabilities in Stock Market Action. Econometrica, 28, pp. 909-915.

Engle, R. F. (1982). Autoregressive Conditicnal Heteroskedasticity with Estimates of the Variance of UK Inflation. Econometrica, 50, pp. 987-1008.

Fama, E. F. (1972). Efficient Capital Markets: a Review of the Theory and Empirical Work. In Lorie, J. (Ed.), Modern Developments in Investment Management. New York.

Kendall, M. J. (1953). The Analysis of Economic Time Series. Part I: Prices. Journal of the Royal Statistical Society, Series A, 96, pp. 11-25.

Loughran, T., and J. R. Ritter (2000). Uniformly Least Powerful Tests of Market Efficiency. Joumal of Financial Economics, (55)3, pp. 361-389.

Ludlow, J. (1997). Modelos, Pronósticos y Volatilidad de las Series de Tiempo Generadas en la Bolsa Mexicana de Valores. UAM-Azcapotzalco. México.

Mills, T.C. (1999). The Econometric Modelling of Financial Time Series. 2nd ed. Cambriedge University Press, Cambriedge.

Moctezuma, A. (1998). Análisis del nivel de eficiencia del mercado accionario mexicano (1989-1997). CNBV Revista de Bancos y Mercados Financieros, segundo semestre (septiembre), pp. 3-17.

Poon, S. H., and S. J. Taylor (1992). Stock Returns and Volatility: an Empirical Study of the UK Stock Market. Journal of Banking and Finance, 16, pp. 37-59.

Working, H. (1960). Note on the Correlation of First Differences of Averages in a Random Chain. Econometrica, 28, pp. 916-918. 